\title{
Correction
}

\section{Correction: de Salazar et al. Oxidative Stress in Endurance Cycling Is Reduced Dose-Dependently after One Month of Re-Esterified DHA Supplementation. Antioxidants 2020, 9, 1145}

\author{
Lydia de Salazar ${ }^{1}{ }^{(0)}$, Carlos Contreras ${ }^{2}$, Antonio Torregrosa-García ${ }^{1, * \mathbb{D}}$, Antonio J. Luque-Rubia ${ }^{1}{ }^{(\mathbb{D}}$, \\ Vicente Ávila-Gandía ${ }^{1}\left[\right.$, Joan Carles Domingo ${ }^{3} \mathbb{D}$ and Francisco Javier López-Román ${ }^{1,4}$
}

check for updates

Citation: de Salazar, L.; Contreras, C.; Torregrosa-García, A.; Luque-Rubia, A.J.; Ávila-Gandía, V.; Domingo, J.C.; López-Román, F.J. Correction: de Salazar et al. Oxidative Stress in Endurance Cycling Is Reduced Dose-Dependently after One Month of Re-Esterified DHA

Supplementation. Antioxidants 2020, 9, 1145. Antioxidants 2022, 11, 449. https://doi.org/10.3390/antiox 11030449

Received: 16 November 2021 Accepted: 25 January 2022

Published: 24 February 2022

Publisher's Note: MDPI stays neutral with regard to jurisdictional claims in published maps and institutional affiliations.

Copyright: (C) 2022 by the authors. Licensee MDPI, Basel, Switzerland. This article is an open access article distributed under the terms and conditions of the Creative Commons Attribution (CC BY) license (https:// creativecommons.org/licenses/by/ $4.0 /)$.
1 Sports Physiology Department, Faculty of Health Sciences, UCAM Universidad Católica San Antonio de Murcia, 30107 Murcia, Spain; dssflydia@ucam.edu (L.d.S.); ajluque@ucam.edu (A.J.L.-R.); vavila@ucam.edu (V.Á.-G.); jlroman@ucam.edu (F.J.L.-R.)

2 Innova, Health and Sport Institute, 30100 Murcia, Spain; cjcontreras@ucam.edu

3 Department of Biochemistry and Molecular Biomedicine, University of Barcelona, 08007 Barcelona, Spain; jcdomingo@ub.edu

4 Biomedical Research Institute of Murcia (IMIB-Arrixaca), 30120 Murcia, Spain

* Correspondence: atorregrosa@ucam.edu

Carlos Contreras was not included as an author in the published article [1]. The corrected Author Contributions Statement appears here.

The contributions as an author were Investigation and Resources.

The affiliation is Innova, Health and Sport Institute, 30100 Murcia, Spain, and e-mail is cjcontreras@ucam.edu.

The authors apologize for any inconvenience caused and state that the scientific conclusions are unaffected. The original article has been updated.

\section{Reference}

1. de Salazar, L.; Contreras, C.; Torregrosa-García, A.; Luque-Rubia, A.J.; Ávila-Gandía, V.; Domingo, J.C.; López-Román, F.J. Oxidative Stress in Endurance Cycling Is Reduced DoseDependently after One Month of Re-Esterified DHA Supplementation. Antioxidants 2020, 9, 1145. [CrossRef] [PubMed] 